\title{
A MINKOWSKI-STYLE THEOREM FOR FOCAL FUNCTIONS OF COMPACT CONVEX REFLECTORS
}

\author{
VLADIMIR I. OLIKER
}

\begin{abstract}
This paper continues the study of a class of compact convex hypersurfaces in $\mathbf{R}^{n+1}, n \geq 1$, which are boundaries of compact convex bodies obtained by taking the intersection of (solid) confocal paraboloids of revolution. Such hypersurfaces are called reflectors. In $\mathbf{R}^{3}$ reflectors arise naturally in geometrical optics and are used in design of light reflectors and reflector antennas. They are also important in rendering problems in computer graphics.

The notion of a focal function for reflectors plays a central role similar to that of the Minkowski support function for convex bodies. In this paper the basic question of when a given function is a focal function of a convex reflector is answered by establishing necessary and sufficient conditions. In addition, some smoothness properties of reflectors and of the associated directrix hypersurfaces are also etablished.
\end{abstract}

\section{INTRODUCTION}

A convex reflector is a convex hypersurface which is the boundary of a compact convex body in Euclidean space $\mathbf{R}^{n+1}, n \geq 1$, obtained by taking the intersection of a given set of confocal (solid) paraboloids of revolution. Convex reflectors arise naturally as solutions to nonlinear second order elliptic partial differential equations of Monge-Ampère type describing conservation laws in geometrical optics [15], [5], [4]. Numerous optics and electromagnetic applications require solutions of such equations; for example, the most common reflector antennas are designed with the use of optics conservation laws and require determination of reflecting surfaces [15]. In addition, it follows from recent results in [3], [7], [8], [6], [14] that in variational formulations of such problems as problems of Monge-Kantorovich optimal mass transfer, the radial and focal functions of a convex reflector turned out to be the logarithms of the Kantorovich potentials [10]. Convex reflectors are also important in inverse problems of rendering in computer graphics when the scattering characteristics of reflectors are prescribed in advance [12].

These reasons already provide sufficient motivation for a systematic study of this class of convex hypersurfaces. Moreover, the results in [9], [11] and [2] show that convex reflectors are of independent geometric interest and this paper continues the investigations in this direction.

Received by the editors December 1, 2004.

2000 Mathematics Subject Classification. Primary 49K20, 35J65, 78 A05.

The research of the author was partially supported by the National Science Foundation grant DMS-04-05622, the Air Force Office of Scientific Research under contract FA9550-05-C-0058 and by a grant from the Emory University Research Committee. 
We now briefly describe the content and organization of the paper. In order to make the presentation reasonably self-contained, we first review in section 2 the basic definitions and a few facts about convex reflectors. More details are provided in [11], but this paper can be read independently of [11]. A new fact, also shown in this section, is that the support function of a nondegenerate convex reflector is of class $C^{1}$.

In section 3 we study analytic properties of the focal function of a convex reflector. In convexity theory the support function is one of the main tools for studying convex hypersurfaces as any convex body can be described by its support function. The classical theorem of Minkowski asserts that any sublinear function in $\mathbf{R}^{n+1}$ is the support function of a unique convex body [13]. By contrast, a convex reflector is more conveniently described by the focal function which for each $y$ on a unit sphere $\mathbf{S}^{n}$ defines the focal parameter of a paraboloid of revolution with axis $y$ supporting to that reflector. The main result in section 3 is Theorem 8 which gives necessary and sufficient conditions for a function on $\mathbf{S}^{n}$ to be a focal function of a convex reflector. Thus, this theorem can be viewed as a generalization of the result of Minkowski. Our results also imply that sublinearity by itself (of the appropriately extended from $\mathbf{S}^{n}$ to $\mathbf{R}^{n+1}$ focal function) is not sufficient to define a focal function of a convex reflector.

In section 4 we study the directrix hypersurface of the reflector which is (up to rescaling) its pedal hypersurface. In particular, it is shown that the directrix of a convex reflector is a hypersurface of class $C^{1}$. A geometric proof of this fact was given earlier in [11]; the proof presented here is analytic and more simple.

\section{Preliminaries}

In $\mathbf{R}^{n+1}$ fix a Cartesian coordinate system with the origin $\mathcal{O}$ and let $\mathbf{S}^{n}$ be the unit sphere centered at $\mathcal{O}$. Throughout the paper the term paraboloid means a paraboloid of revolution with focus at $\mathcal{O}$ and axis $y \in \mathbf{S}^{n}$ directed towards its opening. Such paraboloid is denoted by $P(y)$. No other kind of paraboloids will be considered. The closed convex subset of $\mathbf{R}^{n+1}$ bounded by $P(y)$ and containing its axis is denoted by $B(y)$.

The polar radius of $P(y)$ is given by

$$
\rho_{y}(x)=\frac{\tilde{p}}{1-\langle x, y\rangle}, x \in \mathbf{S}^{n} \backslash\{y\},
$$

where $\tilde{p}$ is a nonnegative real number called the focal parameter of $P(y)$. When $\tilde{p}=0$ it is assumed that the paraboloid reduces to an infinite ray in direction $y$ emanating from $\mathcal{O}$. Such paraboloid is called degenerate. When $\tilde{p}=\infty$ the paraboloid is called improper. In case of an improper paraboloid, $B(y) \equiv \mathbf{R}^{n+1}$.

Definition 1. Let $\left\{P(y), y \in \mathbf{S}^{n}\right\}$ be a family of confocal paraboloids,

$$
B=\bigcap_{y \in \mathbf{S}^{n}} B(y), \text { and } R=\partial B .
$$

If $B$ is compact and contains interior points (in the topology of $\mathbf{R}^{n+1}$ ), the closed convex hypersurface $R$ is called a convex reflector (with the light source $\mathcal{O}$ ). If $B$ has an empty interior, $R$ is called degenerate. The set of all nondegenerate convex reflectors with the light source at $\mathcal{O}$ is denoted by $\mathcal{R}$. 
Since a unit vector $y$ and focal parameter $\tilde{p}$ define a paraboloid $P(y)$ uniquely, it is convenient to describe families of confocal paraboloids by functions on $\mathbf{S}^{n}$. Obviously, any function $\tilde{p}: \mathbf{S}^{n} \longrightarrow \overline{\mathbf{R}}^{+}$, where $\overline{\mathbf{R}}^{+}=[0, \infty]$, defines a family of confocal paraboloids $\left\{P(y), y \in \mathbf{S}^{n}\right\}$ with corresponding focal parameters $\tilde{p}(y)$. Even if $\tilde{p}$ is defined only on a subset of $\mathbf{S}^{n}$ we extend it to the entire $\mathbf{S}^{n}$ by setting it $=\infty$ outside of that subset. When a convex reflector is generated by a family of confocal paraboloids described by a function on $\mathbf{S}^{n}$ we say that the reflector is generated by that function.

It is clear that any positive function $\tilde{p}(y), y \in \mathbf{S}^{n}$, such that $\tilde{p}\left(y_{1}\right)<\infty$ and $\tilde{p}\left(y_{2}\right)<\infty$ for at least two points, generates a reflector in $\mathcal{R}$.

Definition 2. Let $A$ be an arbitrary set in $\mathbf{R}^{n+1}$. A paraboloid $P(y)$ is called supporting to $A$ if

$$
A \subset B(y) \text { and } P(y) \cap A \neq \emptyset .
$$

The following lemma is obvious.

Lemma 3. Let $R \in \mathcal{R}$ be a reflector defined by a function $\tilde{p}$. Then at every point of $R$ there exists at least one supporting paraboloid from the family of paraboloids defined by function $\tilde{p}$.

Lemma 4 ([11]). Let $R \in \mathcal{R}$. For any $y \in \mathbf{S}^{n}$ there exists a unique paraboloid $P(y)$ (not necessarily from the family defining $R$ ) supporting to $R$.

For a given reflector $R$ the family of its supporting paraboloids $P(y), y \in \mathbf{S}^{n}$, defines $R$ uniquely. However, not every paraboloid from the family defined by a function $\tilde{p}$ is necessarily supporting to $R$. For that reason we introduce the following

Definition 5. Let $R \in \mathcal{R}$. The function $p(y), y \in \mathbf{S}^{n}$, giving the value of the focal parameter for each $P(y)$ which is supporting to $R$, is called the focal function of the reflector $R$.

For $R \in \mathcal{R}$ we denote by $B(R)$ the compact convex body bounded by $R$. Since the origin $\mathcal{O}$ is an interior point of $B(R)$, any reflector in $\mathcal{R}$ is star-shaped relative to $\mathcal{O}$. Because $R$ is also compact, the focal function satisfies the inequalities $0<p<\infty$ on $\mathbf{S}^{n}$.

Using the star-shapedness of $R$ with respect to $\mathcal{O}$ we can describe $R$ as a graph over $\mathbf{S}^{n}$ by setting

$$
\rho(x)=\sup \left\{\lambda \geq 0 \mid \lambda x \in B(R), x \in \mathbf{S}^{n}\right\} .
$$

The function $\rho$ is called the radial function (cf. [13]). Then any point on $R$ is given by

$$
r(x)=\rho(x) x, x \in \mathbf{S}^{n} .
$$

Here $x$ is treated as a point on $\mathbf{S}^{n}$ and as a unit vector in $\mathbf{R}^{n+1}$. It follows at once from the definition of $R$ that

$$
\rho(x)=\inf _{y \in \mathbf{S}^{n}} \frac{p(y)}{1-\langle x, y\rangle} .
$$

It follows from (4) that for any given $y \in \mathbf{S}^{n}$ and all $x \in \mathbf{S}^{n}$

$$
p(y) \geq \rho(x)(1-\langle x, y\rangle) .
$$


Since for each $y \in \mathbf{S}^{n}$ there exists a paraboloid $P(y)$ supporting to $R$, the equality is attained at some $x \in \mathbf{S}^{n}$ and therefore,

$$
p(y)=\sup _{x \in \mathbf{S}^{n}} \rho(x)(1-\langle x, y\rangle), y \in \mathbf{S}^{n} .
$$

The reflector map $\gamma$ defined by a reflector $R$ is a possibly multivalued map $\gamma: \mathbf{S}^{n} \rightarrow \mathbf{S}^{n}$ such that

$$
\gamma(x)=\bigcup_{\left\{P_{x}(y)\right\}}\{y\}
$$

where $y$ is the axis of a paraboloid $P_{x}(y)$ supporting to $R$ at $r(x)$ and the union is taken over all such paraboloids. Note that by Lemma 4 the map $\gamma: \mathbf{S}^{n} \rightarrow \mathbf{S}^{n}$ is "onto" for any $R \in \mathcal{R}$.

It follows from (4) and (5) that the reflector map can be defined alternatively as

$$
\gamma(x)=\left\{y \in \mathbf{S}^{n} \mid p(y)=\rho(x)(1-\langle x, y\rangle)\right\}, x \in \mathbf{S}^{n} .
$$

If the reflector $R$ is a $C^{1}$ hypersurface and $u$ its outward unit normal field, then

$$
y=\gamma(x)=x-2\langle x, u(x)\rangle u(x),
$$

which is the law of reflection. In this case, the supporting paraboloid at $r(x)=$ $\rho(x) x$ is unique and it is tangent to the reflector $R$ at $r(x)$.

When $R$ is not smooth, (8) is still satisfied at the point $r(x) \in R$ with any of the supporting paraboloids at $r(x)$. The normal $u$ in this case is the normal to a supporting paraboloid at $r(x)$. Here, and everywhere below, it is assumed that the normals to any of the supporting paraboloids are directed outward relative to the convex sets bounded by these paraboloids.

In order to fix the terminology recall that a support function $f$ of a nonempty closed convex body $K \subset \mathbf{R}^{n+1}$ is defined by

$$
f(U)=\sup _{X \in K}\{\langle X, U\rangle\}, U \in \mathbf{R}^{n+1} .
$$

Since $f$ is positively homogeneous, that is,

$$
f(\lambda U)=\lambda f(U) \forall \lambda \geq 0 \text { and } \forall U \in \mathbf{R}^{n+1},
$$

it is completely defined by its values on $\mathbf{S}^{n}$. In the following, to indicate that we are considering the restriction of the support function to $\mathbf{S}^{n}$, we denote its argument by a small letter and refer to it as the support function of the convex hypersurface $\partial K$.

Theorem 6. The support function $h$ of a reflector $R \in \mathcal{R}$ is positive and of class $C^{1}\left(\mathbf{S}^{n}\right)$. The reflector $R$ can be represented as

$$
X(u)=h(u) u+\nabla h(u), u \in \mathbf{S}^{n},
$$

where $\nabla$ denotes the gradient in the standard metric of $\mathbf{S}^{n}$.

To prove this theorem we will need the following

Lemma 7. Let $R \in \mathcal{R}, \rho$ its radial function, and $h(u), u \in \mathbf{S}^{n}$, its support function. For a fixed $u \in \mathbf{S}^{n}$ consider the supporting hyperplane to $R$

$$
\alpha(u)=\left\{Z \in \mathbf{R}^{n+1} \mid\langle Z, u\rangle=h(u)\right\}
$$

and let $C_{u}=R \cap \alpha(u)$. Then there exists only one $x \in \mathbf{S}^{n}$ such that

$$
C_{u}=\{\rho(x) x\} .
$$


Furthermore, there exists no more than one paraboloid $P$ supporting to $R$ for which $\alpha(u)$ is the tangent hyperplane at $\{\rho(x) x\}$.

Proof. Fix some $u \in \mathbf{S}^{n}$ and let $X \in C_{u}$. Let $P_{X}$ be a paraboloid supporting to $R$ at $X$ and $T P_{X}$ the tangent hyperplane to $P_{X}$ at $X$. Since $T P_{X} \cap P_{X}=\{X\}$, we have $T P_{X} \cap R=\{X\}$, that is, $T P_{X}$ is also a supporting hyperplane to $R$ at $X$. Thus, any hyperplane tangent to a supporting paraboloid may contain at most one point of $R$. If $T P_{X}=\alpha(u)$, then (10) is clearly true.

Suppose, $T P_{X} \neq \alpha(u)$ and let $X_{1} \in C_{u}, X_{1} \neq X$. Since $R$ is a boundary of a compact convex body, the segment $X X_{1}: X_{t}=(1-t) X+t X_{1}, t \in[0,1]$, is contained in $C_{u}$. Clearly, for any $t \in(0,1)$ there exists no paraboloid supporting to $R$ at $X_{t}$, because for any such paraboloid its tangent hyperplane at $X_{t}$ will contain the segment $X X_{1}$, which is impossible. Thus, $R \cap \alpha(u)=\{X\}$ and this implies (10).

The last statement of the lemma follows essentially from the preceding part. Indeed, for any paraboloid $P$ supporting to $R$ at $X$ and such that the tangent hyperplane at the point of support is $\alpha(u)$, the contact set $R \cap \alpha(u)=\{X\}$. Let $x=X /|X|$. By reflection law (8) the axis of $P$ is defined uniquely as

$$
y=x-2\langle x, u\rangle u .
$$

Since the focus of $P$ is fixed at $\mathcal{O}$, it follows from (1) that the vectors $x, y$ define $P$ up to a homothety with respect to $O$. But $P$ also contains $X$, and therefore it is defined uniquely.

Proof of Theorem 6. By Lemma 7, for any $u \in \mathbf{S}^{n}$ the contact set $C_{u}=R \cap \alpha(u)$ consists of only one point. Now, except for positivity of $h$, all statements of this theorem follow from Corollary 1.7.3 in [13], p. 40.

The positivity of $h$ follows from the fact that the origin $\mathcal{O}$ is strictly inside the convex body bounded by $R$.

\section{A Minkowski-TyPe THEOREM FOR FOCAL FUnCTIONS}

The main goal of this section is to determine conditions under which a given function on $\mathbf{S}^{n}$ is the focal function of a convex reflector in $\mathcal{R}$. The following theorem answers this question.

Theorem 8. Let $R \in \mathcal{R}$ and let $p$ be the focal function of $R$. Then $p$ is a positive bounded function on $\mathbf{S}^{n}$ and if for $x, y \in \mathbf{S}^{n}$

$$
x-y=\sum_{i=1}^{k} \alpha_{i}\left(x-y_{i}\right), \quad \alpha_{i} \geq 0, \sum_{i=1}^{k} \alpha_{i}>0,
$$

where the vectors $\left\{x-y_{1}, \ldots, x-y_{k}\right\}$ are linearly independent, then the inequality

$$
p(y) \leq \sum_{i=1}^{k} \alpha_{i} p\left(y_{i}\right)
$$

is satisfied.

Conversely, let $p$ be a positive bounded function on $\mathbf{S}^{n}$ such that for $x, y \in \mathbf{S}^{n}$ satisfying (11) the inequality (12) holds. Then there exists a unique reflector $R \in \mathcal{R}$ with the focal function $p$. 
We shall need the following

Lemma 9. Let $R \in \mathcal{R}, y_{0} \in \mathbf{S}^{n}$, and $P\left(y_{0}\right)$ a paraboloid supporting to $R$ at some point $X$. Let $x=X /|X|$. Then the following decomposition holds:

$$
x-y_{0}=\sum_{i=1}^{k} \alpha_{i}\left(x-y_{i}\right), \alpha_{i} \geq 0, \sum_{i=1}^{k} \alpha_{i}>0,
$$

where $y_{i}$ are the axes of paraboloids supporting to $R$ at $X$, vectors $x-y_{1}, \ldots, x-y_{k}$ are linearly independent and $k \leq n+1$.

Remark 10. The case when $y_{0}$ coincides with one of the vectors $y_{1}, \ldots, y_{k}$ is not excluded. In such case there is only one term in the sum and the corresponding coefficient is equal to one.

Proof. Consider the set of rays originating at $X$ and intersecting $R$ at points different from $X$. The closure of this set (as a set of directions on a unit sphere centered at $X$ ) we denote by $\Psi_{X}$ and its boundary by $\psi_{X}$. Because $\mathcal{O}$ is an interior point of $B(R)$, the cone $\Psi_{X}$ has a nonempty interior. It is also convex. The rays in $\psi_{X}$ form the tangent cone to $R$ at $X$.

Let $\Phi_{X}$ be the cone with vertex $X$ dual to $\Psi_{X}$. We denote its boundary by $\phi_{X}$. It is a cone dual to $\psi_{X}$. Because $\Psi_{X}$ has a nonempty interior and $\Phi_{X}$ is convex, it is clear that there exists a hyperplane $Q$ passing through $X$ for which $Q \cap \Phi_{X}=\{X\}$. Let $Q^{+}$be the halfspace determined by $Q$ which contains $\Phi_{X}$ and $Q_{1}$ the hyperplane parallel to $Q$, at a distance 1 from $Q$, in the halfspace $Q^{+}$. The intersection $\Pi=Q_{1} \cap \Phi_{X}$ is a compact convex set on $Q_{1}$. Its boundary $\pi=Q_{1} \cap \phi_{X}$.

Observe also, that because $\mathcal{O}$ is in the interior of $B(R)$, Theorem 6 implies that for all hyperplanes supporting to $R$ at $X$ we have

$$
\langle x, u\rangle=\frac{h(u)}{|X|} \geq c_{0}=\text { const }>0,
$$

where $u$ is the outward normal to such a hyperplane and $c_{0}$ is independent on a particular supporting hyperplane at $X$.

Now let $P\left(y_{0}\right)$ be a supporting paraboloid at $X$. It follows from (8) applied to $P\left(y_{0}\right)$ that $x-y_{0}$ is orthogonal to the hyperplane tangent to $P\left(y_{0}\right)$ at $X$ and therefore the ray originating at $X$ in direction $x-y_{0}$ is in the set $\Phi_{X}$. Let $v_{0}$ denote the point of intersection of this ray with $Q_{1}$. Since $\Pi$ is the convex hull of $\pi$ and $v_{0} \in \Pi$, by Carathéodory's theorem we have,

$$
v_{0}=\sum_{i=1}^{k} \beta_{i} v_{i}, \beta_{i} \geq 0, \sum_{i=1}^{k} \beta_{i}=1, v_{i} \in \pi,
$$

where $v_{1}, \ldots, v_{k}$ are affinely independent as points of $Q_{1}$ (considered as a Euclidean space) and $k \leq n+1$.

On the other hand, each $v_{i} \in \pi$ is a point on the ray orthogonal to some hyperplane containing a ray tangent to $R$ at $X$. Such hyperplane is also tangent to some paraboloid $P\left(y_{i}\right)$ supporting to $R$ at $X$. Therefore, for some $\gamma_{i}>0$

$$
v_{i}=X+\gamma_{i}\left(x-y_{i}\right)=X+2 \gamma_{i}\left\langle x, u_{i}\right\rangle u_{i},
$$

where $u_{i}$ is the outward unit normal to the hyperplane tangent to $P\left(y_{i}\right)$ at $X$. In addition, by (14), $\gamma_{i}<\infty$. Similarly, for some $\gamma_{0}, 0<\gamma_{0}<\infty, v_{0}=X+\gamma_{0}\left(x-y_{0}\right)$. 
This together with (15) implies (13) with $\alpha_{i}=\beta_{i} \gamma_{i} / \gamma_{0} \geq 0$. It also follows from (15) that

$$
\sum_{i=1}^{k} \alpha_{i}>0
$$

Finally, the linear independence of vectors $\gamma_{1}\left(x-y_{1}\right), \ldots, \gamma_{k}\left(x-y_{k}\right)$ in $\mathbf{R}^{n+1}$ follows from affine independence of points $v_{1}, \ldots, v_{k}$ in $Q_{1}$.

Proof of Theorem 8. Necessity. Let $R \in \mathcal{R}$ and let $p(y), y \in \mathbf{S}^{n}$, be the focal function of $R$. The boundedness of $p$ follows from compactness of $R$ and $p>0$ on $\mathbf{S}^{n}$ because $\mathcal{O}$ is an interior point of $B(R)$ and by (5).

We show now that the inequalities (12) are satisfied. Indeed, let $X$ be an arbitrary point on $R, x=X /|X|$ and $P(y)$ a paraboloid supporting to $R$ at $X$. Then, taking into account (11), we have

$$
p(y)=|X|\langle x-y, x\rangle=\sum_{i} \alpha_{i}\left(|X|\left\langle x-y_{i}, x\right\rangle\right) .
$$

By Lemma 4 for each $y_{i}$ there exists a supporting paraboloid $P\left(y_{i}\right)$. By definition of the focal function the focal parameters of $P\left(y_{i}\right)$ are $p\left(y_{i}\right)$ and, since $P(y)$ is supporting at $X$, we have by (4)

$$
|X| \leq \frac{p\left(y_{i}\right)}{\left\langle x-y_{i}, x\right\rangle}
$$

Then

$$
p(y) \leq \sum_{i} \alpha_{i} p\left(y_{i}\right)
$$

This completes the proof of necessity.

Sufficiency. Since $p$ is positive and bounded, we can define the reflector $R$ as

$$
R=\partial\left(\bigcap_{y \in \mathbf{S}^{n}} B(y)\right)
$$

where $B(y)$ is the closed convex body bounded by paraboloid $P(y)$ with focal parameter $p(y)$. Obviously, $R \in \mathcal{R}$. We need to show that for each $y \in \mathbf{S}^{n} p(y)$ is a focal parameter of a paraboloid supporting to $R$.

Fix any such $y$ and suppose the corresponding paraboloid is not supporting. By construction, $R \subset B(y)$ and, since $P(y)$ is not supporting to $R, R \cap P(y)=\emptyset$. By Lemma 4 there exists a unique paraboloid $\bar{P}(y)$ supporting to $R$ at some $X \in R$. Let $\bar{p}(y)$ be the focal parameter of $\bar{P}(y)$. Obviously, $\bar{p}(y)<p(y)$.

By Lemma 9 we have the decomposition

$$
x-y=\sum_{i=1}^{k} \alpha_{i}\left(x-y_{i}\right), \alpha_{i} \geq 0, \sum_{i=1}^{k} \alpha_{i}>0, \text { and } k \leq n+1,
$$

where $x=X /|X|$ and $y_{i}$ are the axes of paraboloids supporting to $R$ at $X$ and not all of $\alpha_{i}$ are zeroes. Then

$$
\bar{p}(y)=|X|\langle x-y, x\rangle=\sum_{i=1}^{k} \alpha_{i}|X|\left\langle x-y_{i}, x\right\rangle=\sum_{i=1}^{k} \alpha_{i} p\left(y_{i}\right)<p(y),
$$

which contradicts (12). 
It is of interest to compare Lemma 9 with the well-known result for convex hypersurfaces which states that if $M$ is a convex hypersurface, $Q$ is a supporting hyperplane to $M$ at $X$ and $u$ is the outward unit normal to $Q$, then there exist $\alpha_{i}, i=1, \ldots, k \leq n+1, \alpha_{i} \geq 0$, such that the decomposition

$$
u=\sum_{i=1}^{k} \alpha_{i} u_{i}
$$

holds with $u_{1}, \ldots, u_{k}$ being linearly independent outward normals to hyperplanes supporting to $M$ at $X$ [1], p. 40. The seemingly natural extension of this result to convex reflectors with normals replaced by the axes of supporting paraboloids is not possible. This can be seen from the following example.

Let $P_{1}=P\left(y_{1}\right), P_{2}=P\left(y_{2}\right)$ be two confocal paraboloids in $\mathbf{R}^{3}$, with axes $y_{1}$ and $y_{2}$, respectively, common focus $\mathcal{O}$ and positive focal parameters. Assume that $y_{1}$ and $y_{2}$ are perpendicular to each other. Let $R_{2}$ be the convex reflector formed by $P_{1}$ and $P_{2}$. Consider a point $X \in P_{1} \cap P_{2}$ not lying in the plane spanned by $y_{1}$ and $y_{2}$. The axes of paraboloids which are supporting to $R_{2}$ at $X$ lie on a circular cone with axis parallel to the line supporting to $P_{1} \cap P_{2}$ at $X$. Any such axis different from $y_{1}$ and $y_{2}$ cannot be represented as a linear combination of $y_{1}$ and $y_{2}$.

In the rest of this section we consider an extension of a focal function to the entire $\mathbf{R}^{n+1}$ and give a quantitative expression for its "deviation" from a subadditive function.

Recall that a function $f: \mathbf{R}^{n+1} \longrightarrow \mathbf{R}$ is sublinear if it is positively homogeneous and subadditive. The latter means that

$$
f\left(X+X^{\prime}\right) \leq f(X)+f\left(X^{\prime}\right) \forall X, X^{\prime} \in \mathbf{R}^{n+1} .
$$

A well-known theorem of Minkowski asserts that sublinearity is a necessary and sufficient condition for a function in $\mathbf{R}^{n+1}$ to be the support function of a unique compact convex body [13], p. 38 .

Let $R \in \mathcal{R}$ and let $p$ be its focal function. It follows from the definition (2) of the radial function $\rho$ of $R$ and from the expression (5) for its focal function $p$, that $p$ can be naturally extended to the entire $\mathbf{R}^{n+1}$ by setting

$$
p(Y)=\sup _{X \in B(R)}(|X||Y|-\langle X, Y\rangle), Y \in \mathbf{R}^{n+1} .
$$

The extended $p$ is obviously positively homogeneous and it is shown in [11] that it is also subadditive. Then, by Minkowski's theorem, it is the support function of a compact convex body in $\mathbf{R}^{n+1}$. It will be shown in the next section (Theorem 14) that the boundary of this body is the directrix hypersurface (defined in the next section) of the reflector $R$.

The next proposition shows that focal functions of convex reflectors satisfy an inequality more restrictive than the one defining subadditivity.

Proposition 11. Let $R \in \mathcal{R}$ and let $p(y), y \in \mathbf{S}^{n}$, be its focal function. Extend $p$ to the entire $\mathbf{R}^{n+1}$ as in (16). Then for any $Y_{1}, \ldots, Y_{N} \in \mathbf{R}^{n+1}$ and $\alpha_{i} \geq 0, i=$ $1, \ldots, N$, the extended focal function of $R$ satisfies the inequality

$$
p\left(\sum_{i=1}^{N} \alpha_{i} Y_{i}\right) \leq \sum_{i=1}^{N} \alpha_{i} p\left(Y_{i}\right)+|X|\left(\left|\sum_{i=1}^{N} \alpha_{i} Y_{i}\right|-\sum_{i=1}^{N} \alpha_{i}\left|Y_{i}\right|\right),
$$


where $X$ is a point on $R$ at which the paraboloid with axis $\sum_{i=1}^{N} \alpha_{i} Y_{i}$ is supporting to $R$. It is assumed here that if $\sum_{i=1}^{N} \alpha_{i} Y_{i}=\mathcal{O}$, then $X=\mathcal{O}$.

Proof. First, note that the right hand side in (17) is always nonnegative. Indeed, using (16), we obtain

$$
\begin{array}{r}
\sum_{i=1}^{N} \alpha_{i} p\left(Y_{i}\right)+|X|\left(\left|\sum_{i=1}^{N} \alpha_{i} Y_{i}\right|-\sum_{i=1}^{N} \alpha_{i}\left|Y_{i}\right|\right) \\
=\sum_{i=1}^{N} \alpha_{i} \sup _{Z \in B(R)}\left(|Z|\left|Y_{i}\right|-\left\langle Z, Y_{i}\right\rangle\right)+|X|\left(\left|\sum_{i=1}^{N} \alpha_{i} Y_{i}\right|-\sum_{i=1}^{N} \alpha_{i}\left|Y_{i}\right|\right) \\
\geq \sum_{i=1}^{N} \alpha_{i}\left(|X|\left|Y_{i}\right|-\left\langle X, Y_{i}\right\rangle\right)+|X|\left(\left|\sum_{i=1}^{N} \alpha_{i} Y_{i}\right|-\sum_{i=1}^{N} \alpha_{i}\left|Y_{i}\right|\right) \\
=|X|\left|\sum_{i=1}^{N} \alpha_{i} Y_{i}\right|-\left\langle X, \sum_{i=1}^{N} \alpha_{i} Y_{i}\right\rangle \geq 0 .
\end{array}
$$

Put $Y=\sum_{i=1}^{N} \alpha_{i} Y_{i}$. Then for the paraboloid with axis $Y$ supporting to $R$ at $X$, we have

$$
\begin{array}{r}
p(Y)=|X||Y|-\langle X, Y\rangle=\sum_{i=1}^{N} \alpha_{i}\left(|X|\left|Y_{i}\right|-\left\langle X, Y_{i}\right\rangle\right)+|X|\left(|Y|-\sum_{i=1}^{N} \alpha_{i}\left|Y_{i}\right|\right) \\
\leq \sum_{i=1}^{N} \alpha_{i} p\left(Y_{i}\right)+|X|\left(|Y|-\sum_{i=1}^{N} \alpha_{i}\left|Y_{i}\right|\right) .
\end{array}
$$

Remark 12. Taking $Y=Y_{1}+Y_{2}$ in the above proposition one sees immediately that the extended focal function is subadditive. The proposition also shows that subadditivity by itself is not sufficient for a positive and bounded function to be a focal function of a convex reflector.

Remark 13. If $Y=\sum_{i=1}^{N} \alpha_{i} Y_{i}, \alpha_{i} \leq 0, i=1,2, \ldots, N$, then

$$
p\left(\sum_{i=1}^{N} \alpha_{i} Y_{i}\right) \geq \sum_{i=1}^{N} \alpha_{i} p\left(Y_{i}\right)+|X|\left(\left|\sum_{i=1}^{N} \alpha_{i} Y_{i}\right|-\sum_{i=1}^{N} \alpha_{i}\left|Y_{i}\right|\right) .
$$

The proof is similar to the proof of Proposition 11.

\section{Directrix of A REFleCtor}

Associated with a reflector $R \in \mathcal{R}$ is its directrix hypersurface $D(R)$ defined as follows. Let $r(x)=\rho(x) x, x \in \mathbf{S}^{n}$, be the position vector of the reflector $R$, where $\rho$ is the radial function. For each $x \in \mathbf{S}^{n}$ put

$$
D_{x}(R)=r(x)-\bigcup_{y \in \gamma(x)}\{\rho(x) y\},
$$

where $\gamma$ is the reflector map. The directrix of $R$ is defined as

$$
D(R)=\bigcup_{x \in \mathbf{S}^{n}} D_{x}(R)
$$


The directrix of a reflector was introduced and partially studied in [11]. Theorem 14 below provides additional and more detailed information about $D(R)$.

Theorem 14. Let $R \in \mathcal{R}$. Then:

(1) $D(R)$ is a closed convex hypersurface with the origin $\mathcal{O}$ in the interior of the compact convex body bounded by $D(R)$;

(2) for any paraboloid $P(y)$ supporting to $R$ the vector $-y$ is the outward unit normal to a hyperplane supporting to $D(R)$. The hyperplanes supporting to $D(R)$ are the directrix hyperplanes of paraboloids supporting to $R$;

(3) the support function $H$ of $D(R)$ satisfies the equality

$$
H(-y)=p(y), y \in S^{n},
$$

where $p$ is the focal function of $R$;

(4) $D(R)$ can be parametrized as

$$
r^{D}(u)=2 h(u) u, u \in S^{n},
$$

where $h$ is the support function of $R$. In this parametrization $D(R)$ is of class $C^{1}$.

Proof. Remark 12 together with the positive homogeneity of the focal function extended to $\mathbf{R}^{n+1}$ imply that $p$ is sublinear and, therefore, it is the support function of a unique compact convex body in $\mathbf{R}^{n+1}$. Denote this body by $\mathcal{D}$. Since $p>0$, the origin $\mathcal{O}$ is an interior point of $\mathcal{D}$ and, thus, $\mathcal{D}$ is nondegenerate.

We show now that the boundary of $\mathcal{D}$ is the directrix $D(R)$. Fix some $\bar{y} \in \mathbf{S}^{n}$ and consider the hyperplane

$$
Q(\bar{y})=\left\{Z \in \mathbf{R}^{n+1} \mid\langle Z,-\bar{y}\rangle=p(\bar{y})\right\}
$$

and the halfspace

$$
Q^{-}(\bar{y})=\left\{Z \in \mathbf{R}^{n+1} \mid\langle Z,-\bar{y}\rangle \leq p(\bar{y})\right\} .
$$

We want to show that $Q(\bar{y})$ is supporting to $D(R)$.

Let $P(\bar{y})$ be the paraboloid with axis $\bar{y}$ supporting to $R$. By Lemma 4 such paraboloid exists and is unique. Then

$$
|X|\langle x-\bar{y}, x\rangle \leq p(\bar{y}) \forall X \in B(R)
$$

and the equality is attained only when $X \in R \cap P(\bar{y})$; here, as usual, $x=X /|X|$ for $X \neq \mathcal{O}$. When $X=\mathcal{O}$ this inequality, with any $x \in \mathbf{S}^{n}$, is obvious as $p$ is positive. Since any $Z \in D(R)$ is given by

$$
Z=|X|(x-y)
$$

for some $X \in R$ with $y$ being the axis of a supporting paraboloid at $X$, we have

$$
\langle Z,-\bar{y}\rangle=|X|\langle x-y,-\bar{y}\rangle \leq\langle X,-\bar{y}\rangle+|X| \leq p(\bar{y}),
$$

that is, $D(R) \subset Q^{-}(\bar{y})$.

Let $\bar{X} \in R \cap P(\bar{y})$. Then $|\bar{X}|(\bar{x}-\bar{y}) \in D(R) \cap Q(\bar{y})$, that is, the hyperplane $Q(\bar{y})$ is supporting to $D(R)$. Thus, for each $\bar{y} \in \mathbf{S}^{n}, D(R)$ and $\mathcal{D}$ have the same supporting hyperplane $Q(\bar{y})$. Hence, $\partial \mathcal{D}=D(R)$ and the support function $H(-y)$ of $D(R)$ is $p(y)$. It is also clear that $Q(\bar{y})$ is the directrix hyperplane of $P(y)$. This proves (1)-(3). 
Let us now show that $D(R)$ can be parametrized as in (20). Let $u \in \mathbf{S}^{n}$ and let $\alpha(u)$ be the hyperplane with outward unit normal $u$ supporting to $R$. By Lemma 7 there exists a unique $X(u) \in R$ such that $\alpha(u) \cap R=\{X(u)\}$. Consider a ray of direction $u$ originating at $\mathcal{O}$. Since $D(R)$ is convex and $\mathcal{O}$ is in the interior of the compact convex body bounded by $D(R)$, this ray intersects $D(R)$ in a unique point $Z(u)$ and by definition of $D(R)$

$$
Z(u)=X(u)-|X(u)| y
$$

for some $y \in \gamma(x(u))$. But

$$
u=\frac{Z(u)}{|Z(u)|}=\frac{x(u)-y}{|x(u)-y|} .
$$

By the reflection law (8), applied to paraboloid $P(y)$ supporting to $R$ at $X(u), u$ is the normal vector to $P(y)$ at $X(u)$, that is, the hyperplane $\alpha(u)$ is also the tangent hyperplane to $P(y)$ at $X(u)$. Then,

$$
\langle Z(u), u\rangle=|X(u)|\langle x(u)-y, u\rangle=2|X(u)|\langle x(u), u\rangle=2 h(u) u .
$$

This proves $(20)$.

The $C^{1}$-smoothness of $D(R)$ follows now from Theorem 6 .

Remark 15. The representation (20) shows that the directrix $D(R)$ is a rescaled (with coefficient 2) pedal hypersurface of $R$; cf. [9] and other references there.

\section{REFERENCES}

[1] I. J. Bakelman. Convex Analysis and Nonlinear Geometric Elliptic Equations. SpringerVerlag, Berlin, 1994. MR1305147 (95k:35063)

[2] L. A. Caffarelli, C. Gutierrez, and Qingbo Huang. On the regularity of reflector antennas. Preprint, 2004.

[3] L.A. Caffarelli. Allocation maps with general cost functions. Partial Differential Equations and Applications, 177:29-35, 1996. MR1371577 (97f:49055)

[4] L.A. Caffarelli, S. Kochengin, and V.I. Oliker. On the numerical solution of the problem of reflector design with given far-field scattering data. Contemporary Mathematics, 226:13-32, 1999. MR1660740 (99i:65098)

[5] L.A. Caffarelli and V.I. Oliker. Weak solutions of one inverse problem in geometric optics. Unpublished manuscript, 1994.

[6] W. Gangbo and V. I. Oliker. Existence of optimal maps in the reflector-type problems. ESAIM: Control, Optimization and Calculus of Variations, 13:93-106, 2007. MR2282103

[7] T. Glimm and V.I. Oliker. Optical design of single reflector systems and the MongeKantorovich mass transfer problem. J. of Math. Sciences, 117(3):4096-4108, 2003. MR2027449 (2004k:49101)

[8] T. Glimm and V.I. Oliker. Optical design of two-reflector systems, the Monge-Kantorovich mass transfer problem and Fermat's principle. Indiana Univ. Math. J., 53:1255-1278, 2004. MR2104277 (2005f:49095)

[9] T. Hasanis and D. Koutroufiotis. The characteristic mapping of a reflector. J. of Geometry, 24:131-167, 1985. MR793277 (86j:53004)

[10] L.V. Kantorovich and G.P. Akilov. Functional Analysis, ch. VIII, §4, In Russian. Nauka, Moscow, 1977, 2-nd revised edition. MR0511615 (58:23465)

[11] V.I. Oliker. On the geometry of convex reflectors. PDE's, Submanifolds and Affine Differential Geometry, ed. by B. Opozda, U. Simon and M. Wiehe, Banach Center Publications, 57:155-169, 2002. Errata: Banach Center Publications, v. 69(2005), 269-270. MR1974709 (2004c:53103)

[12] G. Patow and X. Pueyo. A survey of inverse surface design from light transport behavior specification. Computer Graphics Forum, 24:773-789, 2005.

[13] R. Schneider. Convex Bodies. The Brunn-Minkowski Theory. Cambridge Univ. Press, Cambridge, 1993. MR1216521 (94d:52007) 
[14] Xu-Jia Wang. On design of a reflector antenna II. Calculus of Variations and PDE's, 20:329341, 2004. MR2062947 (2005f:78005)

[15] B. S. Westcott. Shaped Reflector Antenna Design. Research Studies Press, Letchworth, UK, 1983.

Department of Mathematics and Computer Science, Emory University, Atlanta, GeORGia 30322-0239

E-mail address: oliker@mathcs.emory.edu 